\title{
METABOLIC PROFILE OF THE TRANSITION PERIOD IN EGYPTIAN BUFFALOES (BULBALUSBULBALIS)
}

\author{
EL-MAGHRABY, M.M. and MAHMOUD, A.E. \\ Department of Veterinary Medicine, Faculty of Veterinary Medicine \\ Suez Canal University, Ismailia, Egypt.
}

Received: 25 May 2016; $\quad$ Accepted: 24 July 2016

\begin{abstract}
The transition period defined as the period from 3 weeks prior to calving to 3 weeks after calving which is considered the most critical phase and the most stressful period of lactation cycle, however there is insufficient available data reflecting changes in this period in buffaloes. The present study aimed to monitor the changes in the metabolic profile during pre and postpartum period of highly producing dairy buffaloes as well as their relationship to herd management. This study was conducted on 10 highly producing Egyptian buffaloes, Ismailia, Egypt. A total number of 110 blood samples were obtained from all animals (eleven samples per each animal) at $-4^{\text {th }},-3^{\text {rd }},-2^{\text {nd }},-1^{\text {st }}$ weeks, at calving time $(0)$ and at $+1^{\text {st }},+2^{\text {nd }},+3^{\text {rd }},+4^{\text {th }},+5^{\text {th }}$ and $+6^{\text {th }}$ week and analyzed for plasma glucose level and serum total proteins, albumin, globulins, urea, triglycerides, total cholesterol, HDL-C, LDL-C, VLDL, BHBA, AST and ALT. The results showed a highly significant increase in serum urea level at calving time and entire postpartum period. Also the glucose level showed a highly significant increase at calving time only and a highly significant decrease in the post-partum period. On the other hand,the later findings were accompanied by a non-significant change of beta-hydroxy butyric acid serum level. The Lipid profile, ALT and AST showed a non-significant changes during the entire periods of study. The results draw attention to explanation of the blood metabolic profile in the assessment of food status of buffaloes and ensuring good health in very demanding physiological states in addition, give some indication that buffaloes are more resistance to post-parturient metabolic disorder.
\end{abstract}

Key words: Metabolic profile, Buffaloes, Transition period.

\section{INTRODUCTION}

The transition period for a dairy cow defined as the period from $3 \mathrm{wk}$. prepartum to $3 \mathrm{wk}$. postpartum (Elliot Block, 2010; Ian and Peter, 2010 and Huawei et al., 2013). This period characterized by physiological, metabolic, and nutritional changes (Drackley, 1999 and Gerardo et al., 2009) due to marked changes in the endocrine status of the animal to support late gestation and the onset of milk synthesis along with a gradual decline in dry matter intake that starts 2-3 weeks prepartum, an abrupt increase in nutrient demand with initiation of lactation results in negative energy balance (Castaneda et al., 2009).

Transition period considers a turning point in the productive cycle of the cow from one lactation to the

Corresponding author: Dr. MAHMOUD, A.E.

E-mail address: ahmed_mostafa@vet.suez.edu.eg

Present address: Department of Veterinary Medicine, Faculty of

Veterinary Medicine, Suez Canal University, Ismailia, Egypt. next (Bauman and Currie 1980). The means in which these changes occur and how they are managed are of great importance as they are closely linked to lactation performance, clinical and subclinical postpartum diseases, and reproductive performance that can significantly affect profitability (LeBlanc et al., 2006).

Water buffalo (Bubalus bubalis) supports a significant share of global milk production and is the major milk producing animal in several countries. They are classified into two subspecies: the river buffalo and the swamp buffalo (FAO, 2016). The water buffalo have the ability to adapt to the hot climates and swampy lands; therefore, water buffaloes have special importance in milk and meat production in the valley of the River Nile in Egypt (Abd Ellah et al., 2013).

The metabolic profile has been reported to provide a method to monitor animal health during this critical period with the main purpose to predict a herd's susceptibility to production diseases (Kevin and Ellen, 2012). The importance of the transition period 
in dairy cattle has been highlighted in several review articles (LeBlanc et al., 2006 and T. Roberts et al., 2012). While, there is lack of the published literatures dealing with this critical period in buffaloes. Therefore, this study was designed tomonitor the changes in the metabolic profile during pre and postpartum period of highly producing dairy buffaloes as well as their relationship to herd management.

\section{MATERIALS and METHODS}

\section{Animals and management}

The present study was carried out in on 10 highly producing Egyptian dairy buffaloes (6 to 7 years old) starting from February 2014 up to June 2014 in Ismailia Governorate, Egypt. The average body condition score (BCS) of buffaloes was $4.03 \pm 0.08$, her average body weight was $476 \pm 5.86 \mathrm{~kg}$ and her average daily milk yield was $12.25 \pm 0.22 \mathrm{~kg}$ per animal. Animals were examined carefully and inspected during this transition period for presence of any abnormal clinical signs and for evidence of any metabolic diseases. Buffaloes during the period of study were kept together under open half shelter system and fed on a mixture of formulated concentrate diet $(15.5 \% \mathrm{CP})$ and roughage, the concentrate consisted of $35 \%$ yellow corn, $12.5 \%$ cotton seed meal, $18 \%$ corn gluten feed $(16 \% \mathrm{CP})$, $15 \%$ soybean meal, $15 \%$ beet, $1.5 \%$ sodium bicarbonate, $1.5 \%$ lime stone and $1.5 \%$ sodium chloride. Rice straw, minerals and vitamin block and tap water were available all time. Buffaloes were fed concentrate diet three times daily and their amount was differ according to her productive status where the amount for late pregnant animal was $8 \mathrm{~kg}$ daily while for early lactation was $15 \mathrm{~kg}$ daily per individual animal, buffaloes were milked automatic three times daily.

\section{Sampling:}

A total number of 110 blood samples were obtained from all animals (eleven samples per each animal) at $4^{\text {th }},-3^{\text {rd }},-2^{\text {nd }},-1^{\text {st }}$ weeks, at calving time $(0)$ and at $+1^{\text {st }},+2^{\text {nd }},+3^{\text {rd }},+4^{\text {th }},+5^{\text {th }}$ and $+6^{\text {th }}$ week. Samples were transported in ice box within 1 hour from collection to the research laboratory at department of Internal Animal Medicine, Faculty of Veterinary Medicine, Suez Canal Universality, Egypt.

Paired blood samples were collected from jugular vein into vacutainer tubes from all buffaloes under the study; the first blood sample was collected in 10 $\mathrm{mL}$ dry, clean and sterilized disposable vacutainer plain tubes and used for obtaining serum and the second blood sample was collected in $3 \mathrm{~mL}$ clean and sterilized disposable vacutainer tubes containing sodium fluoride for estimation of blood glucose level. Samples were allowed to clot for 30 to 60 minutes, then centrifuged at $3000 \mathrm{rpm}$ for 20 minutes and a clear, non-hemolyzed sera were collected and divided into 4 equal parts in eppendorf tubes then stored at $20^{\circ} \mathrm{C}$ until used for the biochemical analysis (Coles, 1986).

\section{Biochemical analysis:}

Estimation of total protein (TP), albumin (ALB), urea, glucose, triglycerides (TG), total cholesterol (TC), high density lipoprotein (HDL-C), low density lipoprotein (LDL-C) concentrations, aspartate amino transferees (AST) activity, alanine aminotransferase (ALT) activity were estimated calorimetrically by using reagent test kits supplied commercially by Diamond company-Egypt, while, Beta-hydroxy butyric acid (BHBA) was measured calorimetrically by using reagent test kits supplied by Ben companyItaly (Young, 2001). Globulins was calculated as the difference between the total protein and albumin. Very low density lipoprotein (VLDL-c) was calculated as one-fifth of the concentration of triglycerides (Friedwald et al., 1972).

\section{Statistical analysis:}

The metabolic profile data were presented as Mean \pm SD and analyzed statistically using one-way analysis of variance (ANOVA); Comparison between pre and post-partum period was carried out using paired T-Test. in all cases, difference were considered significant if $\mathrm{P} \leq 0.05$. All statistical analysis was run through SPSS (version, 20) for windows according to Levesque (2007).

\section{RESULTS}

There is a lack of research worldwide dealing with metabolic profiles of transition period in buffaloes. According to our knowledge, this is one of little studies conducted on buffaloes.

As shown in Table 1, the data of 10 highly producing dairy buffaloes revealed that there were a highly significant increase in the mean value of serum urea level $(\mathrm{P}<0.01)$ and a significant increase in the mean value of total protein $(\mathrm{P} \leq 0.05)$ in postpartum period compared with prepartum period, while there were a non-significant difference in the mean values Albumin. Also a highly significant increase of serum urea level $(\mathrm{P}<0.01)$ at calving time and in postpartum period in this study was recorded (Table 2; Figure 1).

Concerning with plasma glucose concentration, it showed a non- significant change during transition period (Table 1), but a highly significant hyperglycemia $(\mathrm{P}<0.01)$ at calving time only with a highly significant hypoglycemia $(P<0.01)$ in the post-partum period was also observed (Table 2; Figure 2); while serum level of beta-hydroxy butyric acid showed non-significant changes between pre and postpartum periods in addition to a non-significant 
reduction on $2^{\text {nd }}$ week postpartum compared with $5^{\text {th }}$; $6^{\text {th }}$ week postpartum, $1^{\text {st }}$ week prepartum and at calving time. (Tables $1 \& 2$ ).

Lipid profile of the investigated buffaloes during the transition period showed a non-significant changes between pre and postpartum period (Table 1), but there was a significant increase $(P \leq 0.05)$ in both levels of Triglycerides and VLDL on $2^{\text {nd }}$ week postpartum compared with $1^{\text {st }} ; 2^{\text {nd }} ; 3^{\text {rd }}$ week prepartum, $3^{\text {rd }} ; 4^{\text {th }}$ week postpartum and at calving time (Table 2). Both Total cholesterol and HDL-c have the same pattern of change as their levels showed a gradual decline from $4^{\text {th }}$ week prepartum till reach their lowest level at calving time then began to increased again till reached their highest level on $5^{\text {th }}$ week postpartum (Table 2), while LDL-c showed a non-significant difference between all weeks in the pre and post-partum period (Table 2).

Concerning with liver enzyme, the mean values of ALT and AST revealed a non- significant difference between pre and postpartum period and during the transition periods (Table 1).

Table 1: Mean values \pm S.E of metabolic profile at transition period in Egyptian buffaloes.

\begin{tabular}{cccc}
\hline Time of samples & Pre-partum & Post-partum & P-value \\
\hline Analytes & $7.50 \pm 0.17$ & $8.03 \pm 0.13$ & $0.054^{*}$ \\
\hline Total protein (g /dL) & $3.45 \pm 0.06$ & $3.29 \pm 0.09$ & $0.195^{\mathrm{NS}}$ \\
\hline Albumin (g / dL) & $4.04 \pm 0.20$ & $4.74 \pm 0.12$ & $0.794^{\mathrm{NS}}$ \\
\hline Globulin (g / dL) & $0.91 \pm 0.07$ & $0.74 \pm 0.03$ & $0.475^{\mathrm{NS}}$ \\
\hline A / G ratio & $27.05 \pm 1.85$ & $52.33 \pm 4.15$ & $0.001^{* *}$ \\
\hline Urea (mg / dL) & $75.4 \pm 2.45$ & $69.93 \pm 2.45$ & $0.102^{\mathrm{NS}}$ \\
\hline Glucose (mg / dL) & $20.56 \pm 0.46$ & $20.41 \pm 0.59$ & $0.833^{\mathrm{NS}}$ \\
\hline BHBA (mg / dL) & $17.09 \pm 2.53$ & $20.61 \pm 3.39$ & $0.261^{\mathrm{NS}}$ \\
\hline $\begin{array}{c}\text { Triglycerides } \\
\text { (mg / dL) }\end{array}$ & $73.33 \pm 8.34$ & $83.84 \pm 5.66$ & $0.151^{\mathrm{NS}}$ \\
\hline $\begin{array}{c}\text { Total cholesterol } \\
\text { (mg / dL) }\end{array}$ & $49.95 \pm 5.49$ & $55.83 \pm 5.09$ & $0.320^{\mathrm{NS}}$ \\
\hline HDL-c (mg / dL) & $7.55 \pm 0.16$ & $8.06 \pm 0.14$ & $0.103^{\mathrm{NS}}$ \\
\hline LDL-c (mg / dL) & $3.42 \pm 0.51$ & $4.12 \pm 0.68$ & $0.262^{\mathrm{NS}}$ \\
\hline VLDL (mg / dL) & $23.80 \pm 1.56$ & $24.73 \pm 0.78$ & $0.416^{\mathrm{NS}}$ \\
\hline ALT (U / L) & $87.20 \pm 2.84$ & $86.66 \pm 3.62$ & $0.903^{\mathrm{NS}}$ \\
\hline AST (U / L) & &
\end{tabular}

${ }^{\mathrm{NS}}$ : Non-significant $(\mathrm{P}>0.05), *$ : Significant $(\mathrm{P} \leq 0.05) . * *$ : Highly significant $(\mathrm{P}<0.01)$

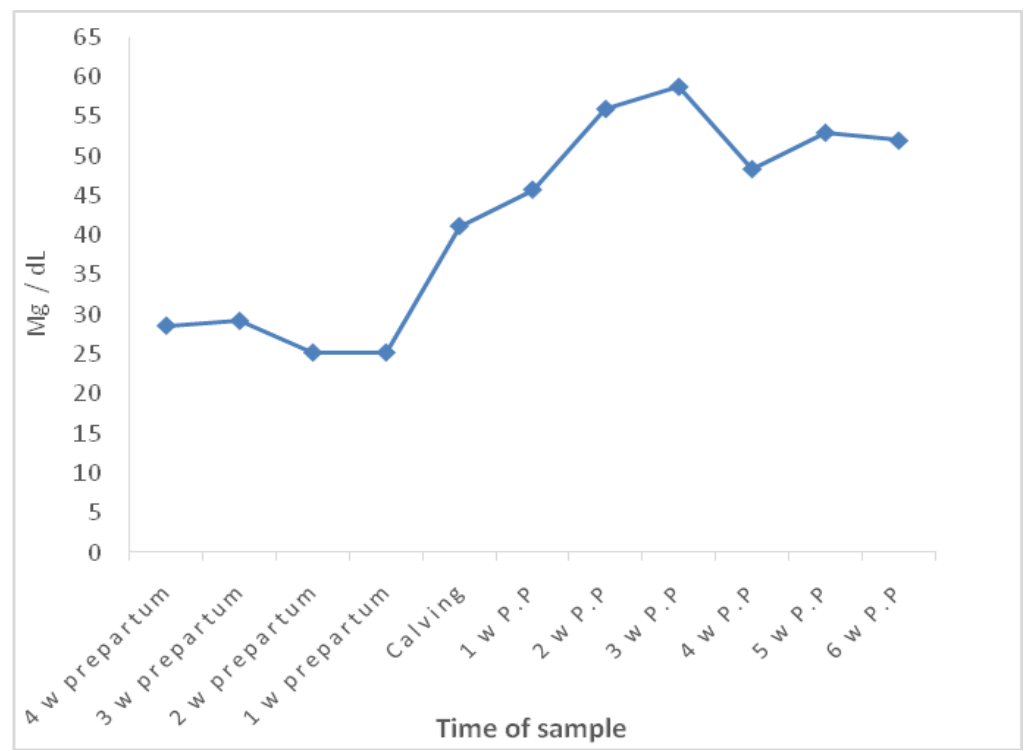

Figure 1: Serum urea level of Egyptian buffaloes during the transition periods. 


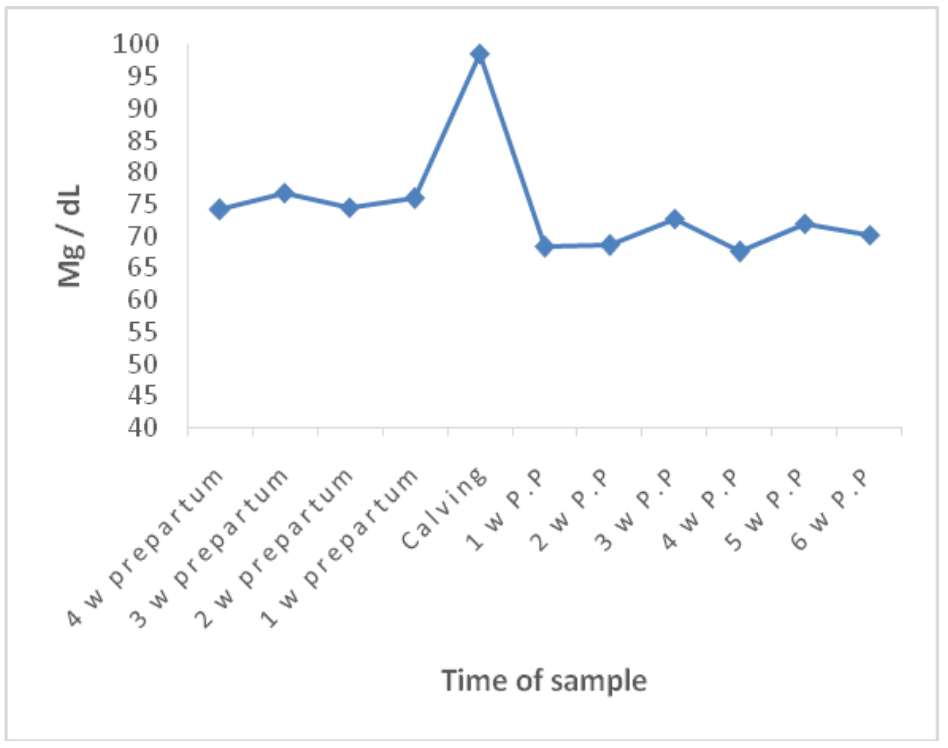

Figure 2: Plasma glucose concentration of Egyptian buffaloes during the transition periods.

Table 2: The mean values \pm S.E of serum metabolic profile during the transition period in Egyptian buffaloes.

\begin{tabular}{|c|c|c|c|c|c|c|c|c|c|c|c|c|}
\hline \multirow{2}{*}{ Analytes } & \multicolumn{11}{|c|}{ Time of samples (weeks) } & \multirow{2}{*}{$\begin{array}{c}\text { P- } \\
\text { value }\end{array}$} \\
\hline & -4 & -3 & -2 & -1 & $\mathbf{0}$ & +1 & +2 & +3 & +4 & +5 & +6 & \\
\hline $\begin{array}{c}\text { Total } \\
\text { protein } \\
(\mathrm{g} / \mathrm{dL})\end{array}$ & $\begin{array}{c}7.6^{\mathrm{a}_{ \pm}} \\
0.22\end{array}$ & $\begin{array}{l}7.6^{\mathrm{a}} \pm \\
0.22\end{array}$ & $\begin{array}{l}7.4^{\mathrm{a}} \pm \\
0.21\end{array}$ & $\begin{array}{c}7.4^{\mathrm{a}} \pm \\
0.35\end{array}$ & $\begin{array}{c}7.4^{\mathrm{a}} \pm \\
0.2\end{array}$ & $\begin{array}{c}7.6^{\mathrm{a}} \pm \\
0.2^{2}\end{array}$ & $\begin{array}{c}7.8^{\mathbf{a}} \pm \\
0.33\end{array}$ & $\begin{array}{c}8.8^{\mathrm{a}} \pm \\
0.8^{-}\end{array}$ & $\begin{array}{c}8.8^{\mathrm{a}} \pm \\
0.9\end{array}$ & $\begin{array}{l}7.8^{\mathbf{a}} \pm \\
0.33\end{array}$ & $\begin{array}{l}7.4^{\mathrm{a}} \pm \\
0.21\end{array}$ & 0.41 \\
\hline $\begin{array}{c}\text { Albumin } \\
(\mathrm{g} / \mathrm{dL})\end{array}$ & $\begin{array}{c}3.7^{\mathrm{a}} \pm \\
0.16\end{array}$ & $\begin{array}{c}3.2^{\mathbf{b c}_{ \pm}} \\
0.08\end{array}$ & $\begin{array}{l}3.4^{\mathrm{abc}} \\
\pm 0.05\end{array}$ & $\begin{array}{c}3.3^{\text {abc }_{ \pm}} \\
0.2\end{array}$ & $\begin{array}{c}3.2^{\text {abc }} \\
\pm 0.1 \\
\end{array}$ & $\begin{array}{l}3.02^{\mathrm{c}} \\
\pm 0.2^{2}\end{array}$ & $\begin{array}{c}3.2^{\text {abc }} \\
\pm 0.1\end{array}$ & $\begin{array}{c}3.2^{\text {abc }} \\
\pm 0.1\end{array}$ & $\begin{array}{c}3.5^{\mathrm{ab}} \pm \\
0.3^{-}\end{array}$ & $\begin{array}{c}3.2^{\text {abc }} \\
\pm 0.1\end{array}$ & $\begin{array}{c}3.5^{\mathrm{ab}} \pm \\
0.14\end{array}$ & 0.18 \\
\hline $\begin{array}{c}\text { Globulin } \\
\text { (g/dL) }\end{array}$ & $\begin{array}{c}3.9^{\mathrm{a}} \pm \\
0.3\end{array}$ & $\begin{array}{c}4.4^{\mathrm{a}} \pm \\
0.2^{2} \\
\end{array}$ & $\begin{array}{c}3.9^{\mathrm{a}} \pm \\
0.2^{2}\end{array}$ & $\begin{array}{c}4.1^{\mathrm{a}} \pm \\
0.5\end{array}$ & $\begin{array}{c}4.3^{\mathrm{a}} \pm \\
0.3 \\
\end{array}$ & $\begin{array}{c}4.6^{\mathrm{a}} \pm \\
0.1 \\
\end{array}$ & $\begin{array}{c}4.6^{\mathrm{a}} \pm \\
0.4 \\
\end{array}$ & $\begin{array}{c}5.5^{\mathrm{a}} \pm \\
0.8\end{array}$ & $\begin{array}{c}5.3^{\mathrm{a}} \pm \\
0.9 \\
\end{array}$ & $\begin{array}{c}4.5^{\mathrm{a}} \pm \\
0.3 \\
\end{array}$ & $\begin{array}{c}3.8^{\mathrm{a}} \pm \\
0.2 \\
\end{array}$ & 0.32 \\
\hline $\begin{array}{c}\text { A / G } \\
\text { ratio }\end{array}$ & $\begin{array}{c}1.01^{\mathrm{a}_{ \pm}} \\
0.12^{\mathrm{n}}\end{array}$ & $\begin{array}{c}0.75^{\mathrm{a}_{ \pm}} \\
0.1 \\
\end{array}$ & $\begin{array}{c}0.91^{\mathrm{a}} \\
\pm 0.06 \\
\end{array}$ & $\begin{array}{c}0.96^{\mathrm{a}} \pm \\
0.2^{2}\end{array}$ & $\begin{array}{c}0.8^{\mathrm{a}_{ \pm}} \\
0.1 \\
\end{array}$ & $\begin{array}{l}0.66^{\mathrm{a}} \\
\pm 0.1 \\
\end{array}$ & $\begin{array}{c}0.69^{\mathrm{a}} \pm \\
0.1 \\
\end{array}$ & $\begin{array}{c}0.64^{\mathrm{a}} \pm \\
0.1 \\
\end{array}$ & $\begin{array}{c}0.78^{\mathrm{a}} \pm \\
0.1 \\
\end{array}$ & $\begin{array}{l}0.73^{\mathrm{a}} \\
\pm 0.1 \\
\end{array}$ & $\begin{array}{l}0.91^{\mathrm{a}} \\
\pm 0.1 \\
\end{array}$ & 0.19 \\
\hline $\begin{array}{c}\text { Urea (mg / } \\
\text { dL) }\end{array}$ & $\begin{array}{c}28.6^{\mathrm{e}} \pm \\
3.21\end{array}$ & $\begin{array}{l}28.9^{\mathrm{e}} \\
\pm 2.40\end{array}$ & $\begin{array}{l}25.2^{\mathrm{e}} \\
\pm 1.07\end{array}$ & $\begin{array}{c}25.2^{\mathrm{e}} \pm \\
2.19\end{array}$ & $\begin{array}{c}41.2^{\text {cd }} \\
\pm 3.2\end{array}$ & $\begin{array}{l}45.8^{\mathbf{b c}} \\
\pm 4.84\end{array}$ & $\begin{array}{c}56^{\mathbf{a b}} \pm \\
5.33\end{array}$ & $\begin{array}{l}58.8^{\mathrm{a}} \\
\pm 4.01\end{array}$ & $\begin{array}{l}48.4^{\text {abc }} \\
\pm 3.56\end{array}$ & $\begin{array}{c}53^{\mathbf{a b c}_{ \pm}} \\
4.84\end{array}$ & $\begin{array}{c}52^{\mathbf{a b c}} \pm \\
5.35\end{array}$ & 0.000 \\
\hline $\begin{array}{l}\text { Glucose } \\
(\mathrm{mg} / \mathrm{dL})\end{array}$ & $\begin{array}{c}74.2^{\mathbf{b}} \pm \\
1.77\end{array}$ & $\begin{array}{c}76.8^{\mathbf{b}} \pm \\
3.87\end{array}$ & $\begin{array}{l}74.6^{\mathbf{b}} \\
\pm 2.32\end{array}$ & $\begin{array}{l}76^{b} \pm \\
8.05\end{array}$ & $\begin{array}{c}98.6^{\mathrm{a}} \pm \\
3.73\end{array}$ & $\begin{array}{c}68.4^{\mathbf{b}} \pm \\
5.23\end{array}$ & $\begin{array}{r}68.6^{\mathbf{b}} \\
\pm 4.12\end{array}$ & $\begin{array}{l}72.8^{\mathbf{b}} \\
\pm 5.07\end{array}$ & $\begin{array}{c}67.6^{\mathbf{b}} \pm \\
3.76\end{array}$ & $\begin{array}{l}72^{\mathbf{b}} \pm \\
3.48\end{array}$ & $\begin{array}{c}70.2^{\mathbf{b}} \pm \\
2.40\end{array}$ & 0.003 \\
\hline $\begin{array}{c}\text { BHBA } \\
(\mathrm{mg} / \mathrm{dL})\end{array}$ & $\begin{array}{c}20.8^{\mathbf{a b c}_{ \pm}} \\
1.17\end{array}$ & $\begin{array}{c}19.4^{\mathbf{b c}_{ \pm}} \\
0.27\end{array}$ & $\begin{array}{c}20.3^{\mathbf{a b c}} \pm \\
0.57\end{array}$ & $\begin{array}{l}21.9^{\mathbf{a b}} \\
\pm 0.77 \\
\end{array}$ & $\begin{array}{c}21.7^{\mathbf{a b}} \\
\pm 0.7\end{array}$ & $\begin{array}{c}20.2^{\mathbf{a b c}} \pm \\
0.29\end{array}$ & $\begin{array}{l}18.2^{\mathrm{c}} \\
\pm 0.84\end{array}$ & $\begin{array}{r}20.2^{\text {abc }} \\
\pm 0.41 \\
\end{array}$ & $\begin{array}{l}19.6^{\mathbf{b c}} \\
\pm 1.12 \\
\end{array}$ & $\begin{array}{l}21.7^{\mathbf{a b}} \\
\pm 0.91\end{array}$ & $\begin{array}{l}22.4^{\mathrm{a}} \\
\pm 1.46 \\
\end{array}$ & 0.10 \\
\hline $\begin{array}{c}\text { Triglyce } \\
\text { rides } \\
(\mathrm{mg} / \mathrm{dL})\end{array}$ & $\begin{array}{c}18.7^{\mathbf{a b}} \pm \\
2.64\end{array}$ & $\begin{array}{r}15.7^{\mathbf{b}} \\
\pm 2.39\end{array}$ & $\begin{array}{l}16.5^{\mathrm{b}} \\
\pm 2.03\end{array}$ & $\begin{array}{c}17.4^{\mathbf{b}_{ \pm}} \\
2.52\end{array}$ & $\begin{array}{l}15.1^{\mathbf{b}} \\
\pm 2.17\end{array}$ & $\begin{array}{l}19.2^{\text {ab }} \\
\pm 1.42\end{array}$ & $\begin{array}{l}27.9^{\mathrm{a}} \\
\pm 4.41\end{array}$ & $\begin{array}{l}18.3^{\mathbf{b}} \\
\pm 2.90\end{array}$ & $\begin{array}{c}15.9^{\mathbf{b}} \pm \\
2.45\end{array}$ & $\begin{array}{l}20.8^{\text {ab }} \\
\pm 3.63\end{array}$ & $\begin{array}{l}21.4^{\text {ab }} \\
\pm 4.45\end{array}$ & 0.30 \\
\hline $\begin{array}{c}\text { T. } \\
\text { Cholesterol } \\
(\mathrm{mg} / \mathrm{dL}) \\
\end{array}$ & $\begin{array}{l}76.5^{\text {abc }} \\
\pm 7.40\end{array}$ & $\begin{array}{c}74.4^{\text {bed }_{ \pm}} \\
7.69\end{array}$ & $\begin{array}{c}73.4^{\text {bcd }} \pm \\
6.61\end{array}$ & $\begin{array}{c}69.1 \text { bcd } \\
\pm 9.45\end{array}$ & $\begin{array}{l}49.7^{d} \\
\pm 8.06\end{array}$ & $\begin{array}{l}59.3^{\text {cd }} \\
\pm 5.26\end{array}$ & $\begin{array}{l}72.2^{\text {bcd }} \\
\pm 2.76\end{array}$ & $\begin{array}{c}79.1^{\text {abc }} \\
\pm 7.8\end{array}$ & $\begin{array}{l}91.5^{\text {ab }} \\
\pm 9.94\end{array}$ & $\begin{array}{l}100.8^{\mathrm{a}} \\
\pm 8.07\end{array}$ & $\begin{array}{l}99.9^{\mathrm{a}} \\
\pm 9.36\end{array}$ & 0.003 \\
\hline $\begin{array}{c}\text { HDL-c } \\
\text { (mg /dL) }\end{array}$ & $\begin{array}{c}52.4{ }^{b^{b}{ }_{ \pm}} \\
4.17\end{array}$ & $\begin{array}{c}50.8^{\mathbf{b c}} \\
6.21\end{array}$ & $\begin{array}{r}49.4^{\text {bcd }} \\
\pm 4.51\end{array}$ & $\begin{array}{c}47.2^{\mathrm{cd}}{ } \\
5.72\end{array}$ & $\begin{array}{l}33.2^{d} \\
\pm 2.93\end{array}$ & $\begin{array}{l}42.6^{\text {cd }} \\
\pm 5.13\end{array}$ & $\begin{array}{l}46.2^{\text {cd }} \\
\pm 3.32\end{array}$ & $\begin{array}{r}53.8^{\mathbf{a b c}} \\
\pm 6.32\end{array}$ & $\begin{array}{r}57.2^{\text {abc }} \\
\pm 6.44\end{array}$ & $\begin{array}{l}69.8^{\mathrm{a}} \\
\pm 7.49\end{array}$ & $\begin{array}{l}65.4^{\mathrm{ab}} \\
\pm 5.75\end{array}$ & 0.009 \\
\hline $\begin{array}{c}\text { LDL-c } \\
(\mathrm{mg} / \mathrm{dL})\end{array}$ & $\begin{array}{c}7.6^{\mathrm{a}} \pm \\
0.22\end{array}$ & $\begin{array}{c}7.8^{\mathrm{a}} \pm \\
0.18\end{array}$ & $\begin{array}{c}7.4^{\mathrm{a}} \pm \\
0.22\end{array}$ & $\begin{array}{c}7.4^{\mathrm{a}} \pm \\
0.36\end{array}$ & $\begin{array}{c}7.6^{\mathrm{a}} \pm \\
0.2^{2}\end{array}$ & $\begin{array}{l}7.6^{\mathrm{a}} \pm \\
0.22\end{array}$ & $\begin{array}{l}7.8^{\mathrm{a}_{ \pm}} \\
0.33\end{array}$ & $\begin{array}{l}8.8^{\mathbf{a}_{ \pm}} \\
0.82\end{array}$ & $\begin{array}{l}8.8^{\mathrm{a}} \pm \\
0.99\end{array}$ & $\begin{array}{l}7.8^{a} \pm \\
0.33\end{array}$ & $\begin{array}{l}7.6^{\mathrm{a}_{ \pm}} \\
0.22\end{array}$ & 0.50 \\
\hline $\begin{array}{c}\text { VLDL } \\
(\mathrm{mg} / \mathrm{dL})\end{array}$ & $\begin{array}{c}3.7^{\mathrm{ab}} \pm \\
0.52\end{array}$ & $\begin{array}{c}3.1^{\mathbf{b}} \pm \\
0.47\end{array}$ & $\begin{array}{c}3.3^{\mathbf{b}} \\
\pm 0.41\end{array}$ & $\begin{array}{c}3.5^{\mathbf{b}} \pm \\
0.51\end{array}$ & $\begin{array}{l}3.1^{\mathbf{b}} \\
\pm 0.4\end{array}$ & $\begin{array}{l}3.8^{\mathbf{a b}} \\
\pm 0.3\end{array}$ & $\begin{array}{c}5.6^{\mathrm{a}} \pm \\
0.88\end{array}$ & $\begin{array}{c}3.6^{\mathbf{b}} \pm \\
0.6^{-}\end{array}$ & $\begin{array}{c}3.2^{\mathbf{b}} \pm \\
0.49\end{array}$ & $\begin{array}{l}4.2^{\mathbf{a b}} \\
\pm 0.7\end{array}$ & $\begin{array}{l}4.3^{\mathbf{a b}} \\
\pm 0.8\end{array}$ & 0.30 \\
\hline $\begin{array}{c}\operatorname{ALT}(\mathbf{U} / \\
\text { L) }\end{array}$ & $\begin{array}{c}23.6^{\mathrm{abc}_{ \pm}} \\
1.87\end{array}$ & $\begin{array}{r}24^{\mathbf{a b c}}{ }_{ \pm} \\
2.15\end{array}$ & $\begin{array}{c}24.2^{\text {abc }} \pm \\
1.11\end{array}$ & $\begin{array}{c}24^{\mathbf{a b c}}{ } \\
1.33\end{array}$ & $\begin{array}{l}21.8^{\mathbf{c}} \\
\pm 0.52\end{array}$ & $\begin{array}{l}21.4^{\mathbf{c}} \\
\pm 0.45\end{array}$ & $\begin{array}{c}24^{\mathrm{abc}_{ \pm}} \\
1.02\end{array}$ & $\begin{array}{r}24.6^{\text {abc }} \\
\pm 1.34\end{array}$ & $\begin{array}{c}25^{\mathrm{abc}_{ \pm}} \\
1.02\end{array}$ & $\begin{array}{l}26^{\text {ab }} \\
\pm 1.69\end{array}$ & $\begin{array}{c}27.4^{\mathrm{a}} \pm \\
0.60\end{array}$ & 0.22 \\
\hline $\begin{array}{c}\operatorname{AST}(\mathbf{U} / \\
\text { L) }\end{array}$ & $\begin{array}{c}77.4^{\mathbf{b}} \\
\pm 3.26\end{array}$ & $\begin{array}{c}90^{\mathbf{a b}} \pm \\
4.15\end{array}$ & $\begin{array}{l}91.2^{\mathrm{a}} \\
\pm 1.96\end{array}$ & $\begin{array}{l}90.2^{\mathbf{a b}} \\
\pm 5.01\end{array}$ & $\begin{array}{c}80.6^{\mathbf{a b}} \\
\pm 2.7\end{array}$ & $\begin{array}{c}90^{\mathbf{a b}} \pm \\
4.49\end{array}$ & $\begin{array}{c}88^{\mathbf{a b}} \pm \\
4.44\end{array}$ & $\begin{array}{l}80.6^{\mathbf{a b}} \\
\pm 4.11\end{array}$ & $\begin{array}{l}85.2^{\text {ab }} \\
\pm 5.03\end{array}$ & $\begin{array}{l}92.8^{\mathrm{a}} \\
\pm 4.18\end{array}$ & $\begin{array}{l}83.4^{\mathbf{a b}} \\
\pm 3.76\end{array}$ & 0.25 \\
\hline
\end{tabular}

Means carrying different superscripts in the same raw are significantly different at $(\mathrm{P} \leq 0.05)$ or highly significantly different at $\mathrm{P}<0.01$ ). 


\section{DISCUSSION}

Analysis of metabolic profile of the investigated buffaloes revealed that there were a highly significant increase in the mean value of serum urea level with a significant increase in the mean value of total protein in postpartum period compared with prepartum period, while there were a non-significant difference in the mean values Albumin. This results was contradicted with data obtained by Neama (2015) in buffaloes however, the later study used animals with different physiological state, but coincided with result of Antunovic et al. (2011) in sheep.

It has been well known that TP, Alb, UREA levels are indicators of sufficient protein intake from diets (Toharmat et al., 1999). Albumin is not a long-term indicator of protein intake because of its relatively short half-life in the blood. On the other hand, UREA level is a good indicator of long term intake of dietary protein (Toharmat et al., 1999 and Kida, 2003), A highly significant increase of serum urea level $(\mathrm{P}<$ 0.01 ) at calving time and in postpartum period in this study can be explained due to excess dietary protein intake in postpartum period which raises the metabolic activity of hepatic microsomes which favors the transformation of alimentary ammonia into urea (Giuseppe et al., 2006).

Concerning with plasma glucose concentration, the increased plasma level of glucose at time of parturition was attributed to the gluconeogenic effect of epinephrine and cortisol because of the excitement and stress associated with parturition (Gerardo et al., 2009), while the recorded hypoglycemia in the postpartum period was attributed to insufficient production of propionate from the low dry matter intake during the early postpartum period (Chandra et al., 2013), also the transient fall in glucose levels in the early stage of lactation can be interpreted due to withdrawal of large amount of blood glucose by the mammary gland for the synthesis of milk lactose (Neama, 2015). Moreover, serum level of betahydroxy butyric acid showed non-significant changes between pre and postpartum periods which is contradicted with the results obtained by (Gerardo et al., 2009) and may be attributed to increase dry matter intake during postpartum which decrease the incidence of negative energy balance (Mark et al., 2004).

The increased concentration of Triglycerides and VLDL on $2^{\text {nd }}$ week postpartum may be attributed to negative energy balance occurred postpartum which leads to increase non-estratified fatty acid (NEFA) which be re-esterified by the liver into triglycerides and exported as very low density lipoproteins (VLDL), an important metabolic response is to mobilize triglycerides (TG's) from adipose tissue to be accumulated in liver (Teama and Gad, 2014) and
(Ghada et al., 2015). These changes did not last long as animal adapteditself, therefore the postpartum fatty liver did not occur and this reflected in the level of BHBA. That is why we had not noticed any significant changes.

The increased concentration of total cholesterol and HDL-c in postpartum weeks was ascribed to lipid catabolism from reserves (Cernescu et al., 2010) furthermore, Neama (2015) reported that the higher level of total cholesterol with advancement of lactation was a physiological adjustment to meet the lactation requirements. Meanwhile the hormonal level of estrogen along with thyroxin played a vital role in reducing the cholesterol levels during pregnancy (Hagawane et al., 2009).

ALT and AST are enzymes that indicate liver cell damage. In this study, the mean levels of both enzymes were non-significantly changed that revealed there was no liver cell damage occurred during transition period (Castaneda-Gutierrez et al., 2009).

\section{CONCLUSION}

The metabolic profile is a beneficial means that has progressed over time. This progression or adaptation is very important to be consider for changes in feeding management and animal genetics. The present data disagree with the most data obtained from cattle in transition period. From these study, it could be concluded that the Egyptian buffalo has the ability to adapt itself on the change of physiological state and therefore it is less susceptible for incidence of post parturient disease. More studies are needed to validate our findings.

\section{REFERENCES}

Abd Ellah, M.R.; Maha I. Hamed; Derar, R.I. and Rateb, H.Z. (2013): Comparative Study on Reference Values for Blood Constituents during Pregnancy in Buffaloes (Bubalusbubalis). Journal of Advanced Veterinary Research Volume 3: 36-46.

Antunovic, Z.; Novoselec, J.; Sauerwein, H.; Speranda, M.; Vegara, M. and Avic, V.P. (2011): Blood metabolic profile and some of hormones concentration in ewes during different physiological status Bulgarian Journal of Agricultural Science, 17 (No 5), 687-695.

Bauman, D.E. and Currie, W.B. (1980): Partitioning of nutrients during pregnancy and lactation: A review of mechanism involving homeostasis and homeorhesis. J. Dairy Sci. 63:1514-1529.

Castaneda-Gutierrez, E.; Pelton, S.H.; Gilbert, R.O. and Butler, W.R. (2009): Effect of peripartum dietary energy supplementation of dairy cows on metabolites, liver function and reproductive 
variables. Animal Reproduction Science, Vol. 112:301-315.

Cernescu, H.; Onitia, P.; Renate Knop; Ionescu, C.; Simona Zarcula and Emilia Groza (2010): The metabolic and hormonal profile on peripartal period in cow. LUCRĂRI ŞTIINTIFICE MEDICINĂ VETERINARĂ VOL. XLIII (2).

Chandra, G.; Aggarwal, A.; Singh, A.K.; Kumar, M. and Upadhyay, R.C. (2013): Effect of Vitamin $E$ and Zinc Supplementation on Energy Metabolites, Lipid Peroxidation, and Milk Production in Peripartum Sahiwal Cows. Asian-Australasian Journal of Animal Scences. Vol. 26, No. 11: 1569-1576.

Coles, E.H. (1986): Veterinary clinical pathology WB Saunders Company. Philadelphia and London.

Drackley, J.K. (1999): Biology of dairy cows during the transition period: the final frontier? Journal of Dairy Science, 82, 2259-2273.

Elliot, B. (2010): Transition Cow Research -What Makes Sense Today? High Plains Dairy Conference, Amarillo, Texas, 76-98.

$F A O$, (2016): Dairy production and products Friedwald, W.T.; Levy, R.T. and Frederickson, D.S. (1972): Estimation of the concentration of low density lipoprotein cholesterol in plasma without use of preparative ultracentrifuge. Clinical chemistry, Vol.18: 499-502.

Ghada A.E. Mohamed; Eman M. Abd-E.naser and Hanan K. Elsayed (2015): Preliminary study on lipid profile with relation to total antioxidant capacity and some hematological and biochemical changes of pre-post-partum buffalo heifers at Assiut city. Assiut Veterinary Medical Journal Vol. 61, No. 144: 159-165.

Gerardo F. Quiroz-Rocha; Stephen J. LeBlanc; Todd F. Duffield; Darren Wood; Ken E. Leslie, and Robert M. Jacobs (2009): Reference limits for biochemical and hematological analytes of dairy cows one week before and one week after parturition. Canadian Veterinary Journal; 50:383-388.

Giuseppe Campanlie; Gianluca Neglia; Rossella Dipalo; Bianca Gasparrini; Corrado Pacelli; Michael J. D'Occhio and Luigi Zicarelli (2006): Relationship of body condition score and blood urea and ammonia to pregnancy in Italian Mediterranean buffaloes. Reproduction Nutrition Development Journal. Vol, 46: 5762.

Ian Lean and Peter De Garis (2010): Transition Cow Management. Dairy Australia.

Hagawane, S.D.; Shinde, S.B. and Rajguru, D.N. (2009): Hematological and Blood Biochemical
Profile in Lactating Buffaloes in and around Parbhani city. Veterinary World, Vol.2, No.12: 467-469.

Huawei Su; Yachun Wang; Qian Zhang; Fuwei Wang; Zhijun Cao; Muhammad Aziz Ur Rahman; Binghai Cao and Shengli Li (2013): Responses of energy balance, physiology, and production for transition dairy cows fed with a low-energy prepartum diet during hot season. Tropical Animal Health Production 45: 14951503.

Kevin Lager and Ellen Jordan (2012): The Metabolic Profile for the Modern Transition Dairy Cow. Mid-South Ruminant Nutrition Conference, Grapevine, Texas.

Kida, K. (2003) Relationships of metabolic profiles to milk production and feeding in dairy cow. J. Vet. Med. Sci. 65:671-677.

LeBlanc, S.J.; Lissemore, D.D.; Kelton, D.F.; Duffield, T.F. and Leslie, K.E. (2006): Major advances in disease prevention in dairy cattle. Journal of dairy science, Vol. 89: 1267-1279.

Levesque, R. (2007): SPSS Programming and Data Management: A Guide for SPSS and SAS Users, Fourth Edition, SPSS Inc., Chicago Ill.

Mark A. McGuire; Mitchell Theurer; John L. Vicini, and Brian Crooker (2004): Controlling Energy Balance in Early Lactation Advances in Dairy Technology (2004) Volume 16, page 241.

Neama A. Ashmawy (2015): Blood metabolic profile and certain hormones concentrations in Egyptian Buffalo during different physiological states. Asian Journal of Animal and Veterinary Advances 10 (6): 271-280.

Teama F.E.I. and Gad, A.E. (2014): Leptin, Thyroxin and Cortisol Hormones and Some Metabolic Products during Pre and Postpartum Periods in Cows in Relations to Their Body Weight of Newborn Calves. Global Veterinaria 12 (1): 59-66.

Roberts, T.; Chapinal, N.; LeBlanc, S.J.; Kelton, D.F.; Dubuc, J. and Duffield, T.F. (2012): Metabolic parameters in transition cows as indicators for early-lactation culling risk. Journal of dairy science Volume 95, Issue 6, Pages 3057-3063.

Toharmat, T.; Nonaka, I.; Shimizu, M. and Kume, S. (1999): Changes of blood composition of periparturient cows in relation to time of day Asian-Aus J. Anim. Sci. vol. 12, No7:11111115.

Young, D.S. (2001): Effect of disease on clinical laboratory test, $5^{\text {th }}$ edition. 


\section{شكل التمثيل الغذائي للفترة الانتقالية فى الجاموس المصرى \\ مدلوح محد المغربى ، /حد السيل محمود}

E-mail: ahmed_mostafa@vet.suez.edu.eg; Assiut University web-site: www.aun.edu.eg

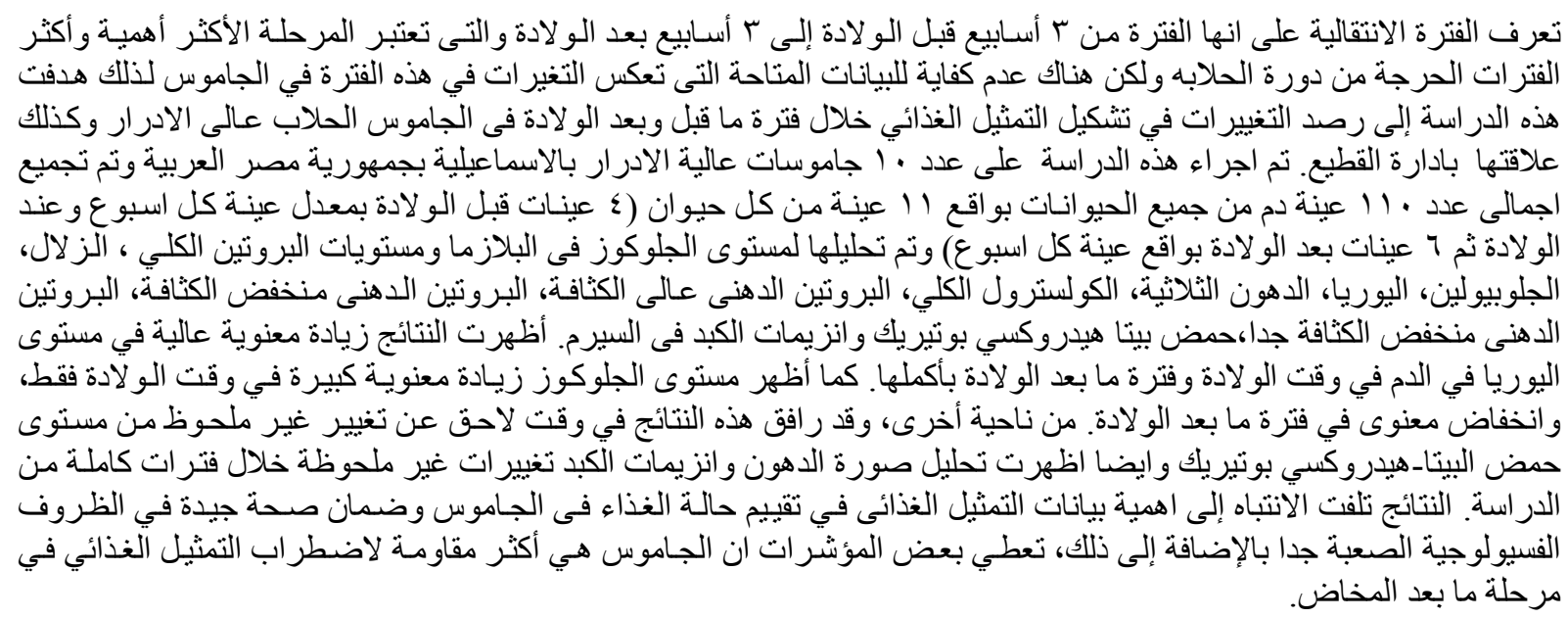

